\title{
Dynamic Neural Network Architectures for on field stochastic calibration of indicative low cost air quality sensing systems
}

\author{
E. Esposito ${ }^{\mathrm{a},}{ }^{*}$, S. De Vito ${ }^{\mathrm{a}}$, M. Salvato ${ }^{\mathrm{a}}$, V. Bright ${ }^{\mathrm{b}}$, R. L. Jones ${ }^{\mathrm{b}}$, O. Popoola ${ }^{\mathrm{b}}$ \\ ${ }^{a}$ UTTP-MDB, ENEA, P.le E. Fermi, 1, 80055 Portici (NA), Italy; \\ (*) Corresponding Author e-mail: elena.esposito@enea.it \\ ${ }^{b}$ Dept. Of Chemistry, University of Cambridge, Lensfield Rd., Cambridge, UK
}

\begin{abstract}
In the last few years, the interest in the development of new pervasive or mobile implementations of air quality multisensor devices has significantly grown. New application opportunities appeared together with new challenges due to limitations in dealing with rapid pollutants concentrations transients both for static and mobile deployments. In this work, we propose a Dynamic Neural Network (DNN) approach to the stochastic prediction of air pollutants concentrations by means of chemical multisensor devices. DNN architectures have been devised and tested in order to tackle the cross sensitivities issues and sensors inherent dynamic limitations. Testing have been performed using an on-field recorded dataset from a pervasive deployment in Cambridge (UK), encompassing several weeks. The results obtained with the dynamic model are compared with the response of the static neural network and the performance analysis indicates the capability of the on-field dynamic multivariate calibration to ameliorate the static calibration approach performance in this real world air quality monitoring scenario. Interestingly, results analysis also suggests that the improvements are more significant when pollutants concentration changes more rapidly.
\end{abstract}

Keywords: Machine Learning, Air quality monitoring, Multivariate Calibration, Dynamic Neural Networks.

\section{Introduction}

Recently, new sensing technologies and systems for achieving a truly pervasive air quality (AQ) monitoring capability in cities are being developed [1]. The primary driver is actually the current coarse grained and sparse AQ measuring mesh that is based on costly and cumbersome conventional analyzers. These are challenged by the need for obtaining a detailed and representative map of the true concentrations of pollutants in the city. As a matter of fact, the current approach is clearly unable to cope with the local complex chemical and fluid dynamic effects occurring in the urban landscape. Emission of air pollutants is caused by different anthropogenic processes which can be categorized into source groups like car traffic, industry, power plants, and domestic fuel. Emitted air pollutants are dispersed and diluted in the atmosphere [2]. Chemical reactions producing, for example, photochemical ozone, occur frequently [3,4]. Dispersion and dilution of air pollutants are strongly influenced by meteorological conditions, especially by wind direction, wind speed, turbulence, and atmospheric stability. Topographical characteristics and urban structures like street canyons, for example, have a significant influence on these meteorological parameters. Eventually, along with chemical reactions, dispersion and dilution processes result in an ambient air pollution distribution which shows concentrations of different substances significantly varying with regard to time and space. Low cost chemical multisensory devices seem a promising answer to the needs; however their performances are hindered by several issues including specificity and stability of transducers. Actually, chemical microsensors devices are, in general, subjected to interferent gases that either boost, or depress, their response, to the target gas [5]. For this reason, any attempt to rely on a monovariate calibration procedure neglecting interferents influence is prone to failure [6]. Information on interferent gases should be exploited by calibration procedure in order to solve this issue. Chemical microsensors response generally changes in time due to several effects including poisoning and 
environmental variables sensitivity [7]. As a consequence, long term stability is a significant concern given the need to reduce maintenance burden on a pervasive network of AQ analyzers.

Although lab based calibration approach allows for fully controlling the range and the ratios of pollutant concentrations to which the sensor array is subject, the exact reproduction of on the field atmosphere is actually precluded by its inherently complex nature. The number of different pollutants and interferents concentrations to consider, in order to cover the experimental space, may easily explode. The use of on-field recorded data may allow solving this issue. On the other hand the concentrations ranges, in this case, are out of the researcher's control. Moreover it could, in principle, reflects a local situation limiting the use of the data for the deployment in different locations. Nonetheless, multivariate calibration with on-field data is being currently reported as an efficient tool [6], [8].

Actually, machine learning (ML) is a promising approach to obtain a multivariate calibration [8]. Flexibility of ML tools can be a decisive asset compared with parametric techniques that require the assumption of a specific hard model form.

Non-linearity in a data set can be detected with graphical methods but identification of its source is more challenging and sometimes impossible. Thanks to their ability to learn and derive input-output relationships from the presentation of a set of training samples, ML tools avoid the time-consuming and possibly expensive task of hard model identification. Generalization properties of ML tools, that is, the capability of a model to produce a valid estimate of the correct output when a totally new input is presented, are a further driver advocating its use with on field recorded data. However, a major drawback is the possibility to overfit calibration data negatively affecting generalization capabilities. Most machine learning can perform at least as well as any other technique in terms of prediction, but a major criticism remains their black-box nature. Model interpretation for a Neural Network, for example, is still considered much more complex than for PLS or PCR models. This is due to the operations (summation and projection on transfer function) performed subsequently in the hidden and output layer, that prevent one from deriving simple analytical expressions between input and output variables.

The usually proposed methodologies, however, are trained to produce instantaneous calibration that do not consider the typically slow and sometimes non-linear (in time) dynamic behaviour of chemical sensors [6] [9]. In our specific scenario this means that significant but relatively short pollution bursts, due for example to moving car or trucks emissions, traffic light related stops, passage of plumes occurring in static deployments or plume crossing by a mobile sensors could be filtered out, masking the real magnitude of the phenomena. This may, in turn, affects time average indicators used for pollution evaluation. Of course these limitations become extremely significant for mobile applications like personal pollution exposition evaluation. In fact, mobile platforms, as they navigate relatively to the source, may cross pollutants plume several times, experiencing sudden and rapid gas concentration transitions [10]. A rapid detection of the concentration changes is hence paramount for personal exposure quantification.

In this view, rapid transient response analysis is extremely relevant. In order to capture the information contained in the dynamics of the gas sensors, artificial olfaction practitioners relies on a fixed, predefined and controlled experimental protocol. Typically, before and after sample presentation, the sensor array is exposed to a gas reference (clean air) to capture the rising and decaying signal transients $[5,11]$. Then, the complete set of acquired time series, or a set of features extracted from the time series [7, 12-15] itself, is used to train a calibration model [16, 17]. In both case, such a calibration methodology requires to capture the sensors signals over a measurement time defined beforehand during a controlled variation of the pollutants concentrations and environmental variables. As a result, the model prediction for a new sample can only be provided after such a measurement is complete. This process, however, is extremely difficult to reproduce when operating in the field requiring complex delivery systems. It is necessary to alternate the gas sample with the reference baseline and the composition of the gas samples has to remain constant, during the whole sample presentation. In open sampling systems the sensor array is exposed directly to the environment with no measurement test chamber, making the system sensitive to flow turbulence [18]. A method that is able to provide continuous and accurate prediction according to the present and past states of the sensor array would be better suited for such applications. Only a few works have explored quantitative prediction algorithms for continuous gas concentration estimation with fast varying concentration inputs. Usually, they were based on a regressor with tapped-delayed input to provide a finite and fixed memory to the system. 
In particular, tapped-delay predictors have been explored with linear (finite impulse response filters) and polynomial regressors (Wiener regressors) [19], neural networks [20-22], or support vector regressors [21].

In [19], S. Marco et al., among the firsts, proposed and compared different nonlinear inverse dynamic models of gas sensing systems for quantitative measurements. With respect to our scenario, a measurement chamber is used to obtain the gas sensor readings, which implicitly modifies the dynamic properties of the measured signals, and the acquisition frequency is too low (one sample per minute) to reflect the fast and highly dynamic changes of the gas concentration in open sampling systems. Anyway, we couldn't find any work based on the use of faster on field analyzer.

In [16], Vergara et al., proposed an approach to accelerate the odor processing using transient features. Recording the response of metal-oxide sensors array, subjected to a specific analyte in a constant flow, they computed the correlation among a transient features and the steady-state resistence. This correlation was used to accelerate standard quantification and classification of analytes.

Again, in [23], the group of D'Amico and S. Marco proposed a so-called ARMA (Auto Regressive Moving Average) system and multi-exponential models, for reducing the time necessary to calibrate a sensor array, taking into account the behaviour of a metal oxide (MOX) semiconductor gas sensor. A dynamic model based on multi-exponential decays allowing a net reduction of the calibration time is introduced and discussed. Nevertheless, since the focus is on the calibration of MOX sensors, the dynamic models are only applied to the rise transient signals recorded in Closed Sampling Systems over long time periods (over 800 s). Moving average and Linear system model identification approaches are compared by Vembu et al. with Support Vector Machines using specific devised time series kernels [42]. Tests have been executed by recording temperature optimized MOX responses in a simulated wind tunnel facility. Results highlighted the performance advantage of the proposed approach.

De Vito et al., in [21], proposed a dynamic calibration based on a tapped delay NN architecture operating on instantaneous and past sensor response samples. In their experimental settings, training samples were obtained by rapidly changing concentrations of multiple gases and environmental conditions. Tests were conducted in the same lab settings confirming the capability of such architectures in improving quantification performance in presence of a slow sensors dynamics. However, in such architectures the memory is fixed by the length of the TD line (or duration of the delay) and has to be optimized in calibration phase. The suitability of such a calibration methodology in a real world settings in which duration and dynamic characteristics of the transient stimulus are not controllable and impredictable is at least controversial and has never been proved.

In $[24,25]$, the authors proposed the use of reservoir computing (RC) algorithms to overcome the slow temporal dynamics of chemical sensor arrays, allowing identification and quantification of chemicals of interest continuously and reducing measurement delays. Moreover, the proposed architecture allows to automatically "learn" the time delay line length and density reducing the needed optimization efforts. The provided results were based on two datasets: one generated with synthetic data and the other acquired from actual gas sensors. They recorded time series of MOX sensors while exposed to binary gas mixtures where concentration levels changed randomly over time.

In the robotics framework, several efforts have been produced for gas mapping and quantitative calibration using partially controlled environments. Recently, J. G. Monroy et al., described an interesting multicentre effort to provide a probabilistic quantification approach based on Gaussian process [41]. They validate the approach within a simulated field environment, in which ethanol is emitted and transported by an air flow towards MOX sensors located in a room. A Photo-Ionization Device (PID) VOC sensor is used as a reference.

Summarizing, to the best of our knowledge, dynamic calibration approaches, known for lab based measurements, has never been validated for on-the-field deployed chemical multisensor devices where they have to deal with the uncontrollable exposure to multiple analyte concentrations over several weeks.

In our opinion, the lack of high speed validated reference measurements is one of the primary factor limiting this development. As a matter of fact, most of the reference stations provide validated readings of hourly concentrations averages limiting validation of high sampling frequency devices. Furthermore, in order to reduce issues and errors related with turbulent flow exposure of both indicative and reference measurement systems, both systems has to analyze the same air almost simultaneously. 
In this contribution, we can analyze the results of a calibrated multisensing device that was co-located with a 1 min sampling frequency conventional analyzer deployed on the field in the city of Cambridge (UK) for several weeks. We propose and test a Dynamic Neural Network approach as a dynamic multivariate calibration tool in a real world deployment, thus extending the results available for static multivariate on-field approaches. Section 2 describes the experimental settings and the results of a preliminary data analysis. Section 3 describes the proposed calibration methodology, whose results are presented in Section 4. Finally, in Section 5, conclusions are drawn.

\section{Experimental Settings}

The Dataset here used has been extracted from a specific deployment of multisensory devices (SnaQ systems, see [26] for detailed description of the deployment) developed by a partnership led by the Center for Atmospheric Sciences (CAAS) of University of Cambridge (UK). The multisensory device is equipped with the following sensors units:

- two different $\mathrm{NO}_{2} \mathrm{EC}$ sensor units (Alphasense $\mathrm{NO}_{2}-\mathrm{B} 4$ termed in the following as $\mathrm{NO}_{2}(\mathrm{~A})$ and $\mathrm{NO} 2(\mathrm{~B})$ );

- one NO Alphasense EC sensor unit (Alphasense NO-B4);

- two different $\mathrm{O}_{3}$ Alphasense EC sensor units (Alphasense $\mathrm{O}_{3}-\mathrm{B} 4$ termed in the following as $\mathrm{O}_{3}(\mathrm{~A})$ and $\mathrm{O}_{3}(\mathrm{~B})$ );

- T, RH sensor units;

- wind speed and direction unit.

One of the devices, deployed in the city Centre of Cambridge, was located on the roof of the Chemical Dept. together with a conventional reference station operated by CAAS. This station relies on chemiluminescence and spectrometer based analyzers. While the sampling period of the SnaQ system was set at 20 seconds, the reference station provides ground truth readings of target gases at $60 \mathrm{sec}$ intervals. The conventional station target gases were $\mathrm{CO}, \mathrm{NO}, \mathrm{NO}_{2}$, $\mathrm{NO}_{\mathrm{x}}, \mathrm{O}_{3}, \mathrm{SO}_{2}$ and Total Oxidants gases. It is worth to note that electrochemical sensors, when operating at low ppb levels, are also prone to interference issues. Specifically for the SnaQ sensors, a known cross sensitivity is reported for, respectively, $\mathrm{O}_{3}$ and $\mathrm{NO}_{2}[27,28]$.

Two different experimental methodologies concerning timeframe harmonization procedures have been considered. A preliminary work has been carried out by averaging three instances of raw sensor readings belonging to the 60 seconds interval before each conventional analyzer sample. In this work, instead we considered all raw instantaneous sensors readings for calibration purposes, comparing the estimations results with the conventional analyzer samples when available (one out of three sensor readings).

Baseline and temperature correction by datasheet procedure have been implemented. Furthermore, a preprocessing step has been conducted to delete reference station calibration periods during the night.

In order to build a suitable dataset, five weeks of continuous measurements have been extracted by the available recordings. The first week of measurements ( 10 ksamples) have been set apart as a training set for statistical machine learning tools, while the remaining four weeks of data have been used for validation (10 ksamples) and testing purposes (30 ksamples). This partition allows for evaluating the performance of the trained model including possible medium term sensors drift effects.

\subsection{Preliminary Data Analysis}

Early data analysis revealed that the monitored zone has significant daily variations of RH and T. In particular, it was frequent to observe daily variation of RH from $20 \%$ to $80 \%$ and temperatures varying from $10^{\circ} \mathrm{C}$ to $25^{\circ} \mathrm{C}$ (see Fig.1, a). Of course this prove challenging for the microsensors array subjected to varying environmental conditions. In the same period, the minimum temperature recorded was less than $8^{\circ} \mathrm{C}$, with the maximum being about $30^{\circ} \mathrm{C}$.

The Roof conventional analyzer dataset (Fig.1, b), generally depicts a high sensitivity of the SnaQ sensors and significant correlation among sensors raw values and target gases, except for $\mathrm{O}_{3}$ sensors (Fig.2, a and Fig.2, b). However, evidence of concept drifts is present (Fig.2, c). Furthermore the relationship among some of the sensors and their target gas seems to show clues of the above mentioned cross sensitivities. Actually in Fig. 3, a it is possible to observe a positive shift in the response of $\mathrm{NO}_{2}(\mathrm{~A})$ sensor, in presence of relatively high NO concentrations. In presence of different concentrations of $\mathrm{O}_{3}, \mathrm{NO}_{2}(\mathrm{~A})$ and $\mathrm{NO}_{2}(\mathrm{~B})$ seems to modify their response very differently. 

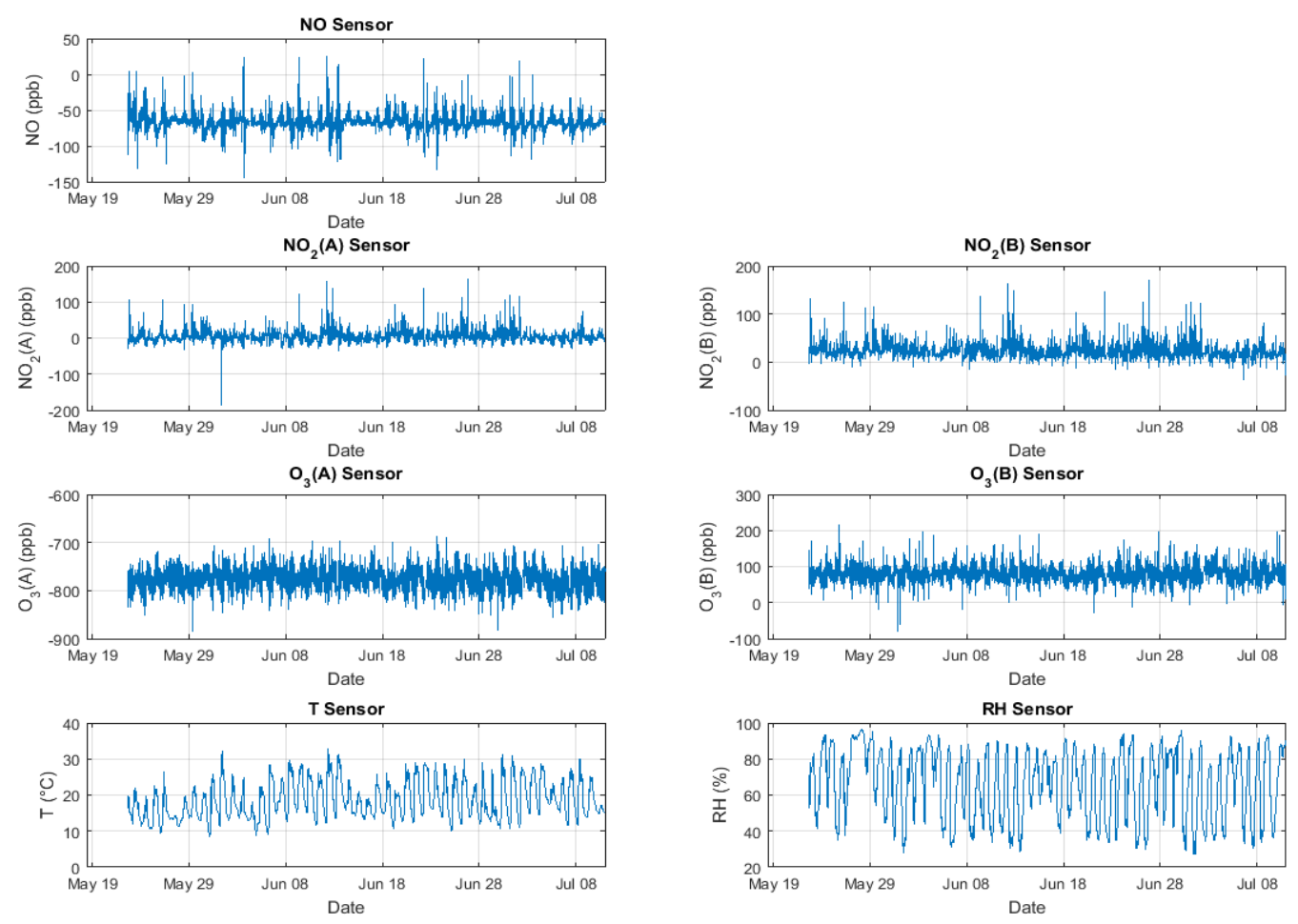

Fig. 1 (a): Plot of uncalibrated SnaQ EC Sensors Data (Temperature Correction apply) versus time (seconds), plus Temperature (T) and Humidity (RH) sensors. The daily oscillation pattern is evident. 

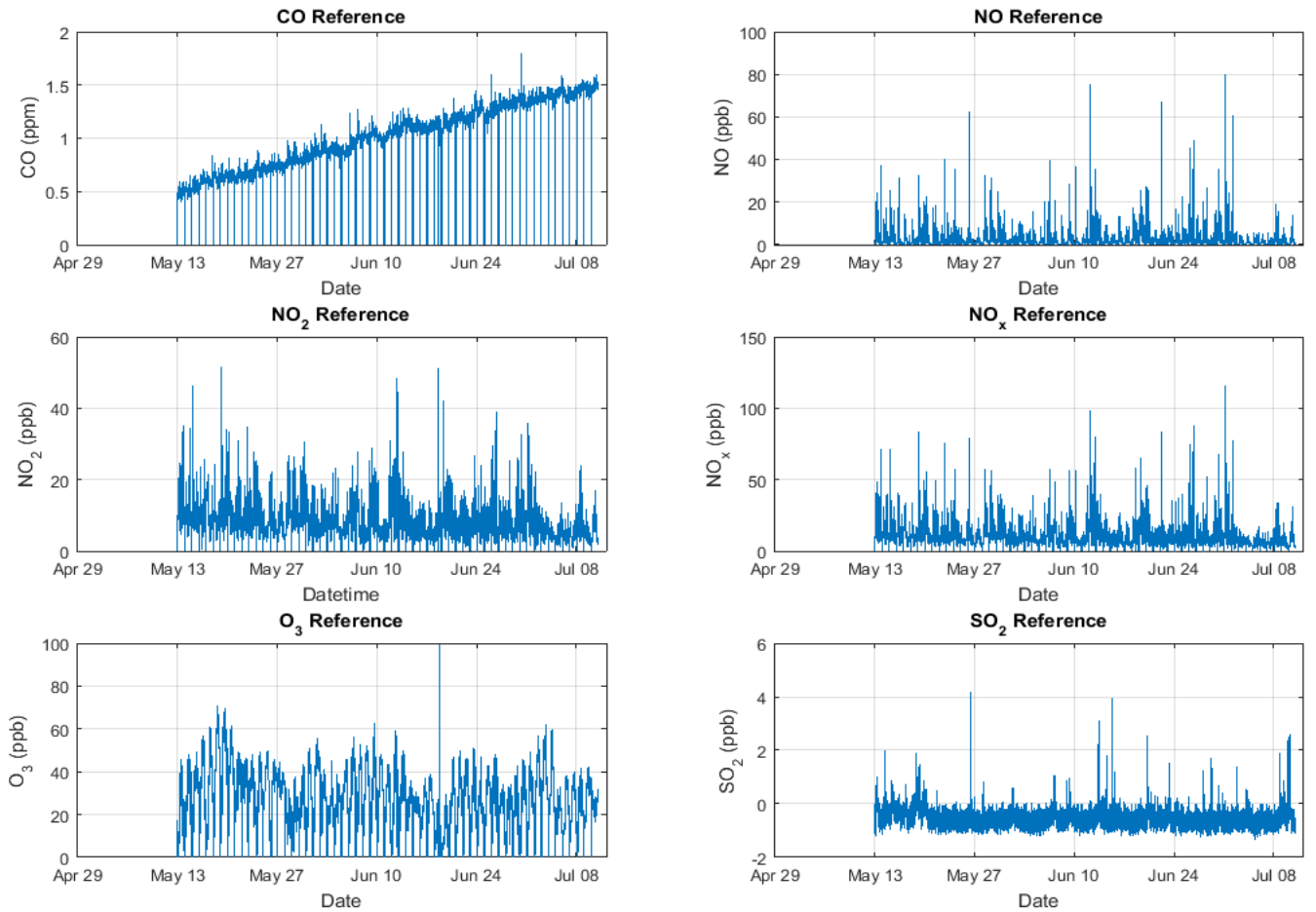

Fig. 1 (b): Plot of reference concentration data (Roof Dataset). The CO concentration data present a linear drift (with) time and it is not used in this work. 

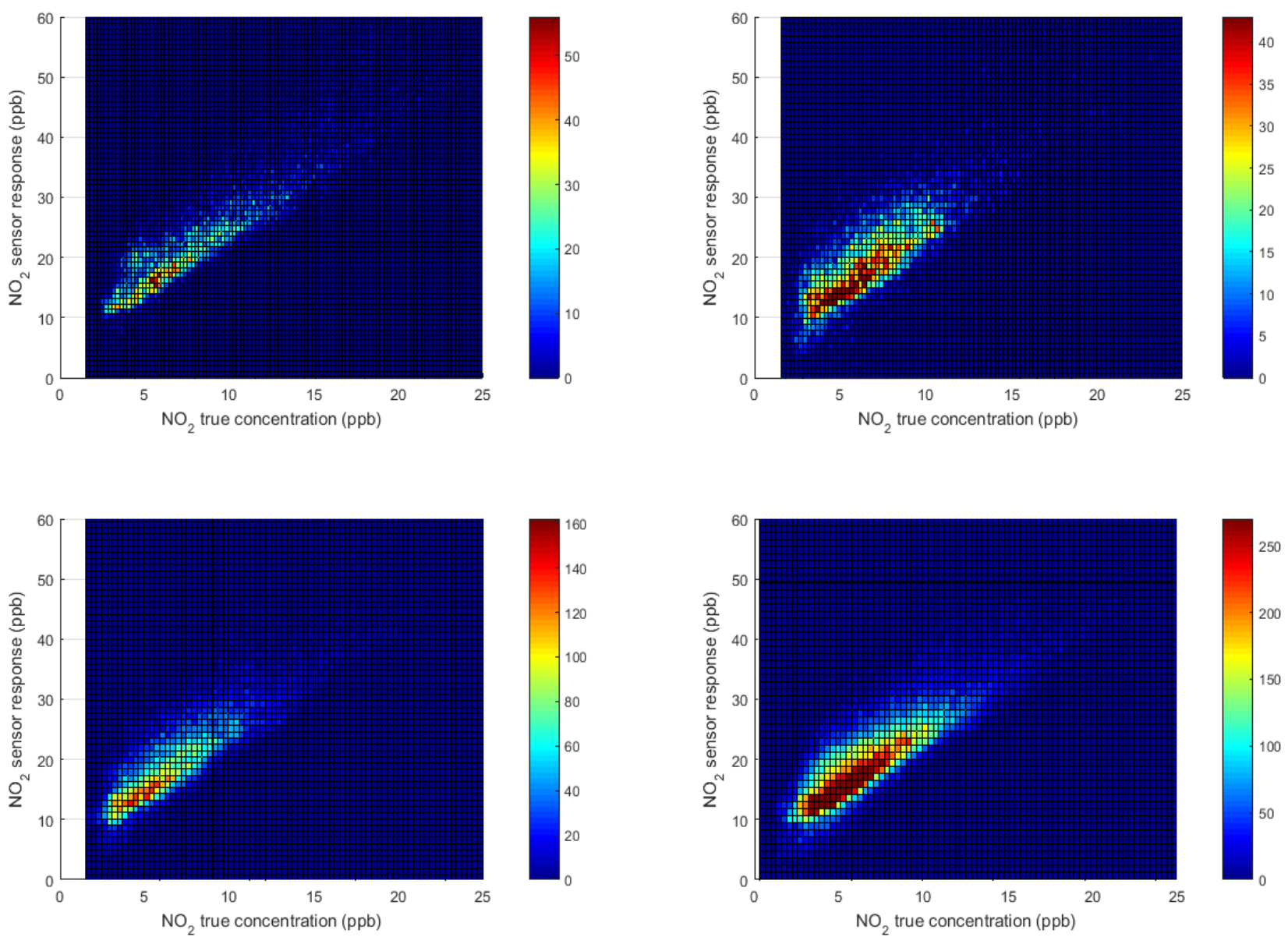

Fig. 2 (a): Data distribution shows significant correlation among target gas concentration and sensor raw values, for $\mathrm{NO}_{2}$ gas concentration (color code is the histogram of the readings). The first figure shows a scatter plot in the first week $(\mathrm{R}=0.88)$, the second, provides the relationship as computed during the fourth week $(R=0.81)$. The third picture relates with third and fourth week, below used as test set $(\mathbf{R}=\mathbf{0 . 8 2})$. Finally, the fourth picture shows correlation plot over the entire dataset $(R=0.85)$. 

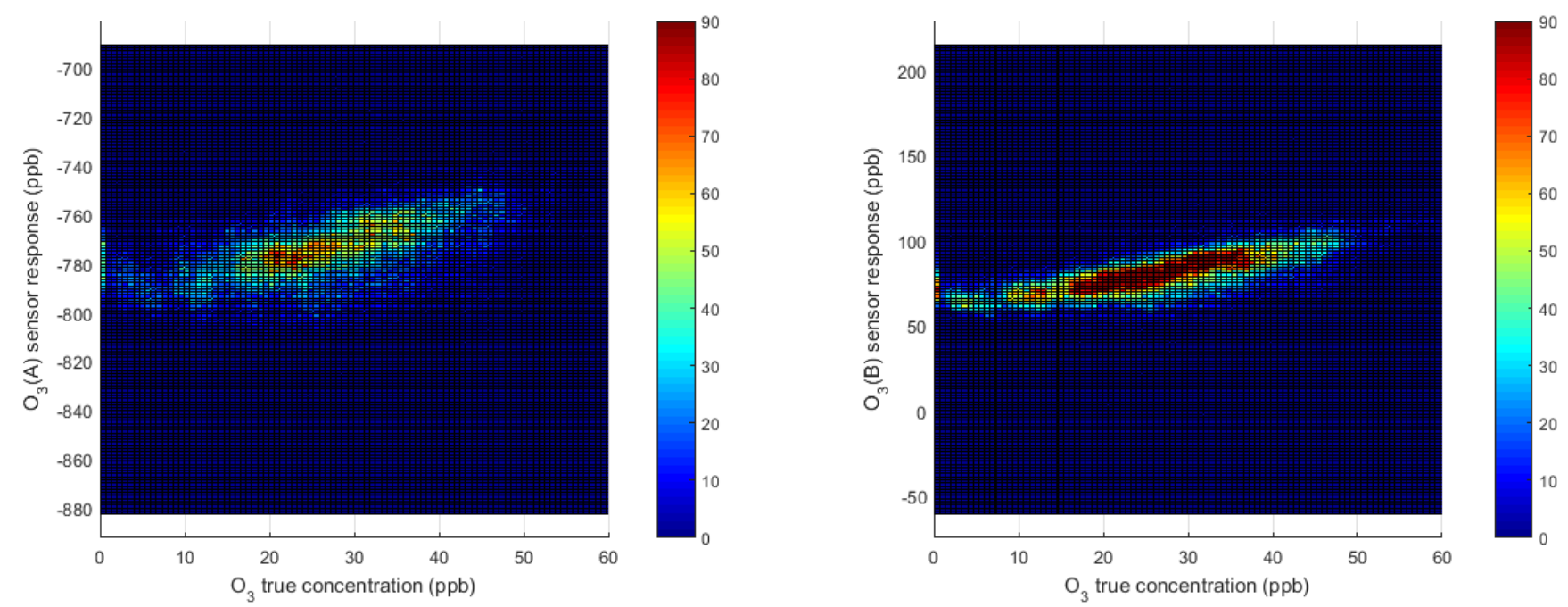

Fig. 2 (b): Data distribution shows inferior correlation among target gas concentration and sensor raw values for $\mathrm{O}_{3}$ gas concentration (color code reflects histogram of the readings). The first figure shows the correlation among target gas and $\mathrm{O}_{3}(\mathrm{~A})$ sensor response $(R=0.51)$, the second, considers $O_{3}(B)$ sensor response $(R=0.67)$.
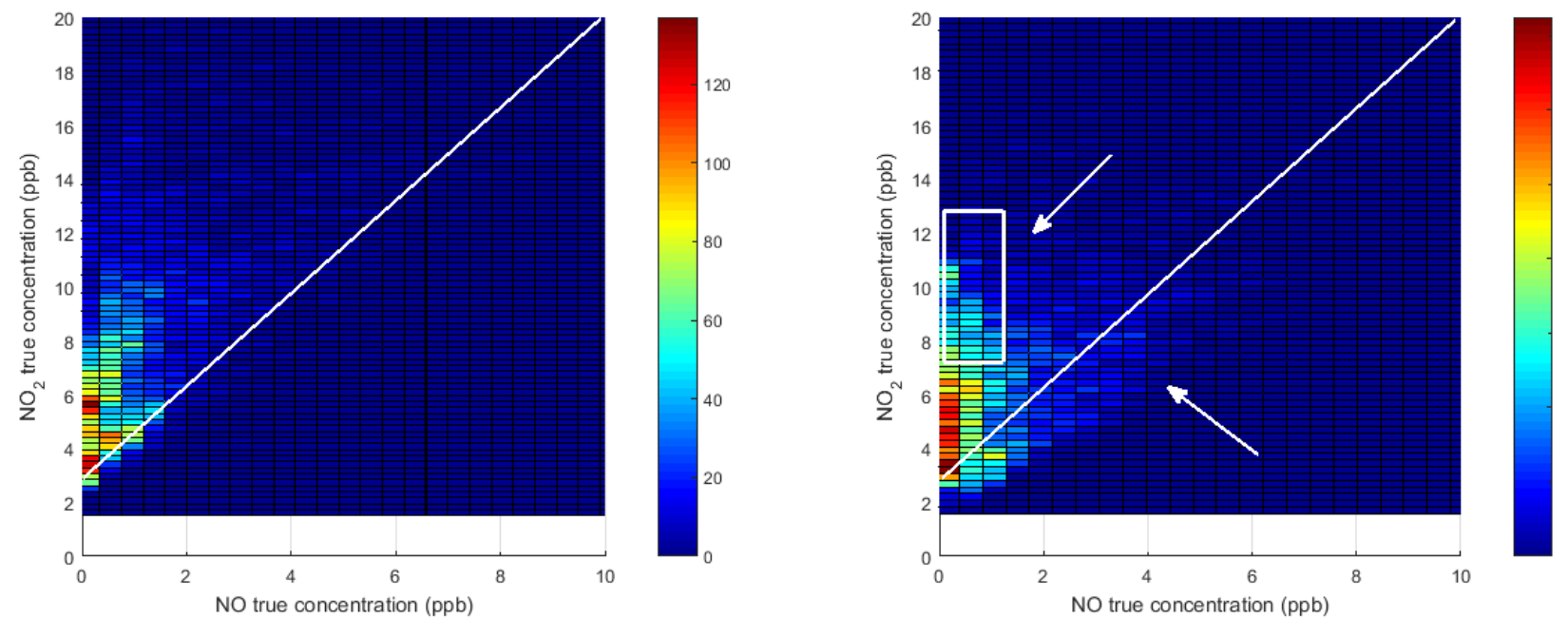

Fig. 2 (c): Mutual distribution of reference data for $\mathrm{NO}$ and $\mathrm{NO}_{2}$. Evidence of complex relationship among the two gases is observed in different weeks with low correlation data $(R=0.55$ in the first and $R=0.52$ in the fourth week). During the fourth week, in particular, the picture shows significant differences with the appearance of a number of occurrences in the two highlighted areas (area inside the white rectangle and below the white line) that were barely populated in the first week. 

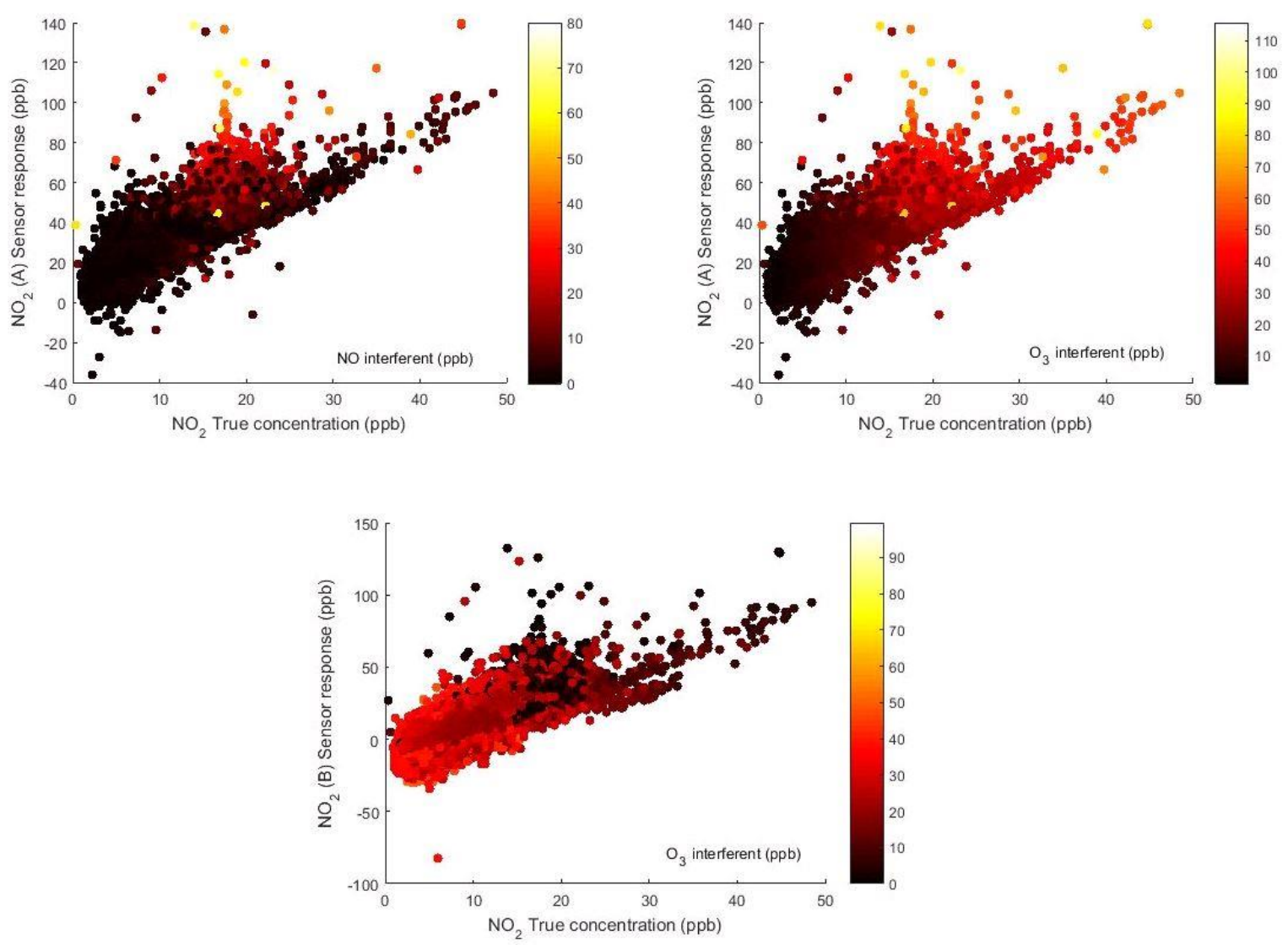

Fig. 3: Scatterplot of $\mathrm{NO}_{2}$ concentration, considering: (a) NO as interferent $\left(\mathrm{NO}_{2}(\mathrm{~A})\right.$ sensor reading); (b) $\mathrm{O}_{3}$ as interferent $\left(\mathrm{O}_{3}(\mathrm{~A})\right.$ sensor reading); (c) $\mathrm{O}_{3}$ as interferent $\left(\mathrm{NO}_{2}(\mathrm{~B})\right.$ and $\mathrm{O}_{3}(\mathrm{~B})$ sensors readings). Dot color encodes the concentration of the interferent gas (ppb).

\section{Dynamic Multivariate Calibration}

The considered multivariate calibration technique includes the use of dynamic and nonlinear supervised machine learning tools. We focused our attention on DNNs in order to exploit their capability to build a dynamic multivariate model of the sensor response. Actually, our aim is to use them to overcome static network limitations in high speed transients evaluation simultaneously tackling sensors cross-specifies. Since, DNNs can be designed to operate as a multivariate regression so to provide pollutant concentration estimations on the basis of raw sensor readings.

In this work, we tested the performance of two particular architectures, comparing the obtained results with the commonly used static Feed Forward Neural Network (FFNN). The first selected dynamic architecture has been a Time Delay Neural Network (TDNN); a Nonlinear Autoregressive with exogenous inputs network (NARX) was the second [30]. DNNs architectures include a tapped delay line that allows using as inputs both past sensor readings and past output values. In this way they can exploit the informative contents of both sensors and process dynamics.

\subsection{Feed Forward Neural Network}

FFNN is the most popular and most widely used neural model in many practical applications. It consists of a (possibly large) number of simple neuron-like processing units, organized in layers. Every unit in a layer is connected with all the units in the previous layer. These connections are not all equal: each connection may have a 
different strength or weight. The weights on these connections encode the knowledge of a network. Often the units in a neural network are also called nodes. The structure of a feedforward neural network is represented in Fig.4, a.

In this work, we considered a FFNN model, with a sigmoid activation function for the neurons in the hidden layer. The network core was based on a Matlab ${ }^{\circledR}$ tansig neurons hidden layer with a single output layer designed to provide estimation of a single pollutant every 1 minute [29]. Model complexity is basically controlled by the number of hidden layer neurons. In our experiments, We have let it vary in the $H N=[3,5,10]$ set.

\subsection{Tapped Delay Neural Network}

TDNN consists of a feedforward network with a tapped delay line at the input [30]. This allows the network to have a finite dynamic response to time series sensors data. In our framework, it can be used to analyze and exploit sensor dynamics. A TDNN is typically described as a layered network in which the input of a layer is buffered for several time steps and then fed to the input layer (see Fig.4, b). By the introduction of $T$ time delays, each neuron has access to $n$ input values, corresponding to different sensors array instantaneous response $x(t-n T), \ldots, x(t)$.

Summarizing the model complexity is controlled by both TD line length $(T D L)$ and the number of hidden layer. In this paper we have let them vary in the $T D L=[3,6,9]$ and $H N=[3,5,10]$ sets, respectively.

\subsection{Nonlinear Autoregressive with Exogenous Inputs Network}

The NARX net is a nonlinear autoregressive neural model which has exogenous inputs. These systems model the dynamic of a variable (time series) as depending on its past values and on the current and past values of external driving input (exogenous inputs).

Practically, the NARX network is a recurrent dynamic network, with feedback connections affecting several layers of the network. The model is based on the linear Autoregressive with Exogenous input (ARX) model, and it is well suited to model nonlinear dynamic systems. It is commonly used in time-series modeling. As for the training of the NARX network, it is noteworthy to highlight that the true output is available during the training of the network. Therefore, this value can be used instead of the estimated output during the training phase. This architecture is illustrated in Fig.4, c. Summarizing the model complexity of our NARX networks is controlled by input delay (TDL) and feedback lines lengths $(F D L)$ and by the number of hidden layer. In this paper we have let them vary in the $T D L=[3,6,9], F D L=[2,5]$ and $H N=[3,5,10]$ sets, respectively. 

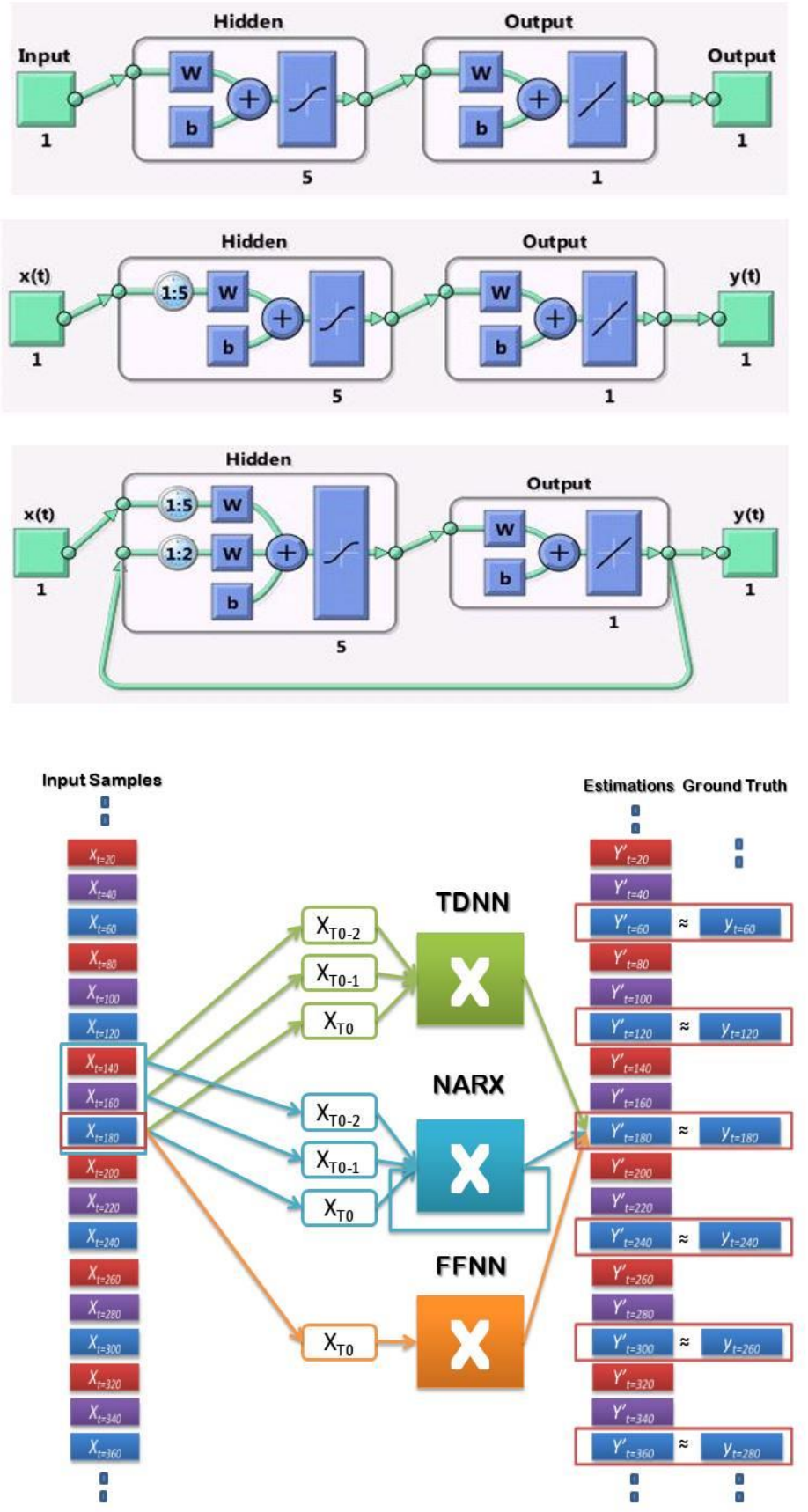

Fig. 4: Relevant examples of FFNN (a), TDNN with $T D L=5$ (b), NARX with $T D L=5, F D L=2$ (c) networks architecture. (d) depicts the modus operandi of the three architectures while estimating $y_{t}$ with $y_{t}^{\prime}$ by using the input samples $x_{t}$ with unfolded timeline. In particular, the depicted TDNN and NARX networks have TDL $=3$ and FDL $=1$. Note that ground truth comparison, and hence performance estimation, is only possible at reference station sample rate $(1 \mathrm{~min})$ while in principle, the networks could estimate pollutant concentration every time a new input sample become available $(20 \mathrm{sec})$. 


\subsection{Performance estimation procedure}

The three above mentioned architecture have been tested for their capability to model and generalize sensors response to target gas concentration relationships. Dataset have been partitioned in training (1 week), validation (1 week) and test set (3 weeks), as described in Section 2. The network training procedure was based on backpropagation with Levenberg-Marquardt algorithm [40]. The validation set have been used for model complexity optimization e.g. selecting the best performing number of hidden layer neurons, tapped delay length and feedback delay line length. Optimization has been conducted by brute force exploration of model complexity spaces, described by model complexity hyperparameters sets, for the three architectures. For this reason, a total number of 30 different NNs architectures (3FFNN+9TDNN+18NARX) have been generated, trained and tested. Mean relative error (MRE) defined as the sample mean of the ratio between absolute prediction error and true concentration value, mean absolute error (MAE) defined as the sample mean of absolute prediction error, Standard Deviation (STD) of the absolute prediction error and correlation coefficient (CC) have been used as performance indicators. Tests set have been used to extract generalization performance indicators. Each training and procedure has been repeated 30 times so to reduce the uncertainty in performance indicators computation induced by the random choice of networks initial weights, by averaging. Best performing networks architectures for FFNN, TDNN and NARX networks, defined by their hyperparameters t-uple, have been selected to be compared.

Obtained differences among indicators have been tested for statistical significance at 0.05 confidence level. Specifically, Two-Sample $t$-test with Unequal Variance for computed MAEs has been used assuming a normal distribution for computed MAEs populations. This $t$-test returns a test decision for the null hypothesis that indicators comes from independent random samples from normal distributions with equal means and unknown and unequal variances. The needed normality assumption have been positively validated using Kolmogorov-Smirnov normality test at 0.01 confidence level for all the obtained MAE estimations (FFNN, TDNN and NARX) over the performed 30 repetitions.

\subsection{Uncertainty Analysis}

Our aim is to provide the sensor node with a methodology to extract locally or remotely precise and accurate target gas concentration estimation, however, the availability of uncertainty estimations will provide definitely more complete information to the user.

Actually, neural networks does not provide a direct methodology for estimation of uncertainty, yet it could be estimated empirically by means of an error distribution estimation procedure, executed using the validation set. To this purpose, by partitioning the relevant values range for target gas in equal intervals $a_{i}$, for each execution of training-validation-test procedure, we have computed the empirical predictive error (err) distribution, given target concentration estimation $y^{\prime}$ :

$$
p\left(\operatorname{err} \mid y^{\prime} \in a_{i}\right)
$$

over the validation samples. In this work, we chose the number of intervals to be 30 .

For each relevant value of $a_{i}$, such empirical distribution has been fitted with a Gaussian model (see Fig. 5). Then, mean $\mu_{a_{i}}^{e r r}$ and standard deviation $\sigma_{a_{i}}^{\text {err }}$ of the fitted distribution have been used to obtain biased uncertainty bars for estimation performed at time $t$, using a coverage factor of $1 \sigma$ :

$$
\mu_{a_{i}, t}^{e r r}-c\left(\sigma_{a_{i}, t}^{e r r}\right) \leq\left(y^{\prime}(\vec{x}, t)-y(t)\right) \leq \mu_{a_{i}, t}^{e r r}+c\left(\sigma_{a_{i}, t}^{e r r}\right)
$$

where $y^{\prime}(\vec{x}, t)$ is the network output, $y(t)$ is the target gas real concentration and $\vec{x}$ is the sensor array instantaneous response.

In this way, the user can be provided by both concentration estimation and prediction uncertainty. To this purpose, in the following, estimation charts have been superimposed with the obtained biased uncertainty bars. 
Finally, the Negative Log Predictive Density (NLPD) measure have been used for testing the performance of obtained confidence intervals estimations. The NLPD, is a regression performance index designed for evaluating uncertainty estimations for generative models or, in general, of every machine learning approaches providing a posteriori conditional distributions. The action of the index is dual inasmuch it both penalize over and underconfident estimations, i.e. estimation with over narrow and over wide a posteriori distributions and their relative confidence intervals spread with respect to actual estimation error magnitudes. Under gaussian assumptions, it can be computed as

$$
N L P D=\frac{1}{2 N}\left\{\sum_{t=1}^{N}\left[\log \left(\text { var }_{t}\right)+\frac{\left(y(t)-\mu_{t}\right)^{2}}{\operatorname{var}_{t}}\right]\right\}+c
$$

where $\operatorname{var}_{t}$ is the predictive a posteriori variance, $\mu_{t}$ is the predictive mean i.e. the expected value of the a posteriori distribution. Constant $c$ is independent of $\mu_{t}$ and var $_{t}$ and here is set to $\frac{\log (2 \pi)}{2}$.

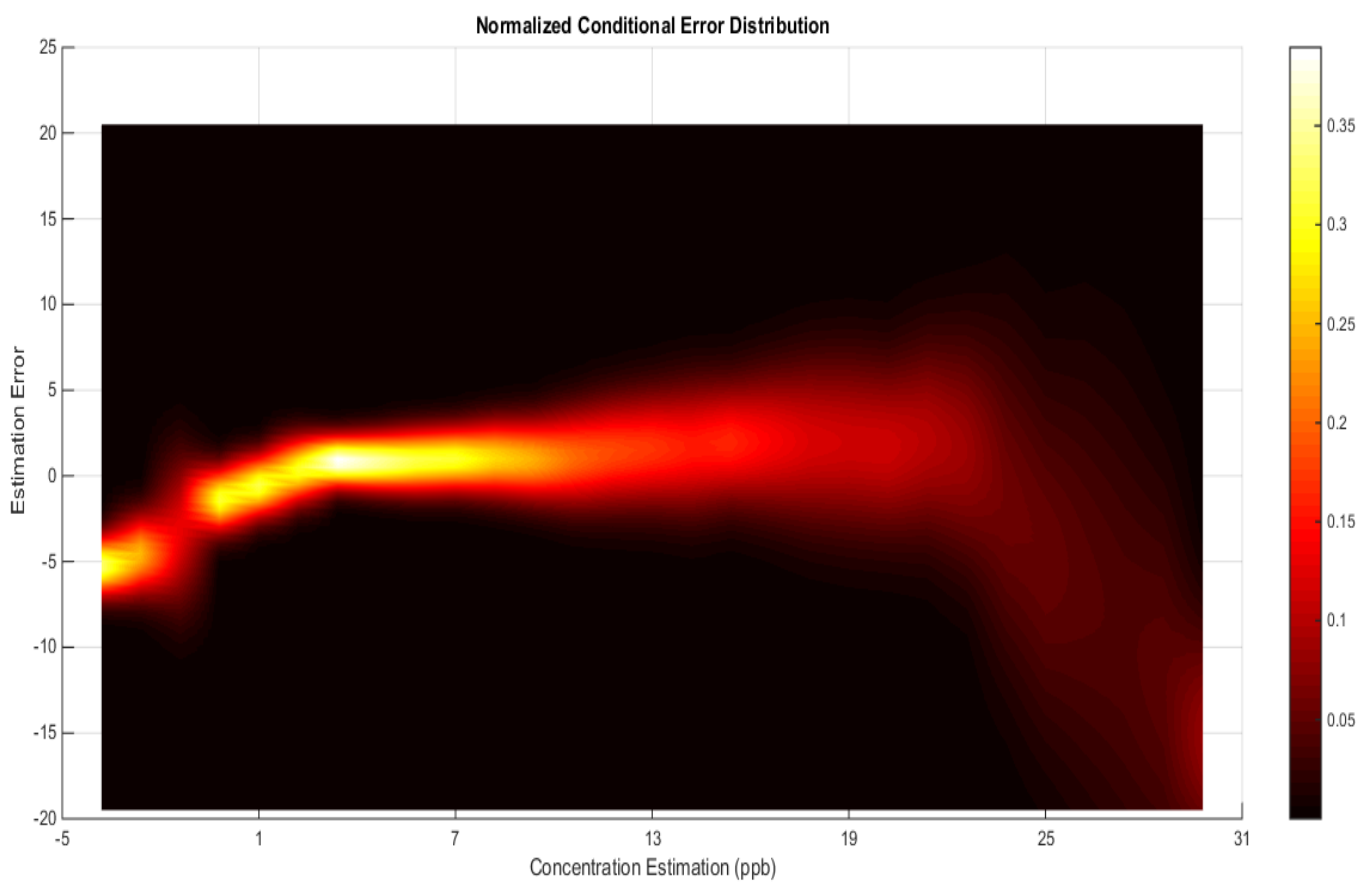

Fig. 5: Example of the normalized empirical conditional error distribution for the TDNN. Probability distribution of error $e=\left(y-y^{\prime}\right)$ given concentration estimation $y^{\prime}$ have been fitted with Gaussian distribution profiles for every interval $a_{i}$. Prediction error bias is non-zero at both the extremes of estimated concentration distribution while standard deviation become visibly wider at high estimated concentration values. Normality assumption have been positively tested with Kolmogorov Smirnov test.

\section{Results}

In this work, we specifically report the results of testing the DNN (TDNN and NARX) architectures with a focus on targeting $\mathrm{NO}_{2}$ concentration estimation comparing them to the results obtained by a static FFNN operating on the same problem with same inputs. We use $\mathrm{NO}, \mathrm{NO}_{2}, \mathrm{O}_{3}, \mathrm{RH}$ and $\mathrm{T}$ high speed sensor response measurements as input variables for filling the delay lines of the tested dynamic architectures ( $T s=20$ secs) while the FFNN have been fed with response samples obtained with $T s=1 \mathrm{~min}$. Error performance for all the architectures have been computed on instantaneous ground truth readings at $T s=1$ minute for which the reference values have been obtained by conventional analyzers station (see fig. 4-d). 
Results of model optimization have allowed the selection of the best performing hyperparameters t-uple for each network class. Surprisingly the best number of hidden layer neurons have been found to be 5 for all the three network classes. For the TDNN networks, the best performing TD length was found to be 6 , so the best TDNN was the $\mathrm{HN}=5$, TDL $=6$ network. Finally, for the NARX class, the best input delay line length was also found at 6 while the feedback delay line for the NARX network was best performing at length 5, so the best NARX network was the $\mathrm{HN}=5$, TDL=6, FDL=5 (see Fig. 6).

\begin{tabular}{|c|c|c|c|}
\hline \multicolumn{4}{|c|}{ Validation Set Performance (MAE) - NARX FDL = 2 } \\
\hline HN & \multicolumn{3}{|c|}{ MAE (pp) } \\
\hline 10 & 1.36 & 1.28 & 1.30 \\
\hline 5 & 1.24 & 1.26 & 1.23 \\
\hline 3 & 1.31 & 1.32 & 1.22 \\
\hline \multicolumn{5}{|c|}{} \\
\hline TDL & 3 & 6 & 9 \\
\hline
\end{tabular}

\begin{tabular}{|c|c|c|c|}
\hline \multicolumn{5}{|c|}{ Validation Set Performance (MAE) - NARX FDL = 5 } \\
\hline HN & \multicolumn{3}{|c|}{ MAp) } \\
\hline 10 & 1.32 & 1.22 & 1.19 \\
\hline $\mathbf{5}$ & 1.30 & $\mathbf{1 . 1 8}$ & 1.27 \\
\hline 3 & 1.26 & 1.33 & 1.25 \\
\hline \multicolumn{5}{|c}{} \\
\hline TDL & 3 & $\mathbf{6}$ & 9 \\
\hline
\end{tabular}

Fig. 6: Validation set MAE averaged on the 30 repetitions as computed for NARX architecture with Feedback delay line length equal to 2 (left) and 5 (right). Bold values indicate the best performing hyperparameters combination.

The resulting best network architectures performances have been selected and compared using results obtained over the test set with the described 30x averaging procedure. In Table 1, we report the results of the comparison tests of the selected models. Results show a significant advantage of the dynamic approach with respect to the static FFNN, with the TDNN emerging as the best performing approach. The results of significance test of the observed differences at 0.05 level confirm this finding. In particular, the obtained $p$ value $\left(<10^{-5}\right)$ allowed sufficient evidence to reject the null hypothesis (equal averaged MAE) for FFNN and NARX computed MAEs comparison. The same, $a$ fortiori, applied for the FFNN and TDNN comparison $\left(\mathrm{p}<10^{-10}\right)$. The test results does not allowed for deeming the observed difference between TDNN and NARX, in the average, as significant at 0.05 level.

The TDNN advantage is best summarized with a percentage gain of $16 \pm 4 \%$ on mean absolute error value over the three weeks test period obtained by FFNN. The NARX advantage is more limited, having been found setting at $13 \pm 5 \%$ over FFNN computed MAE. Furthermore, for TDNN and NARX net, we have obtained a correlation coefficient $r$ equal to 0.91 and 0.88 , respectively, while FFNN reported a $r$ equal to 0.84 . Furthermore, it is worth to note that dynamic networks are also capable to express better confidence interval as witnessed by a lower value of the NLPD loss function.

All this finding collectively support a numerically and statistically significant advantage of the dynamic architectures over static FFNN for the calibration of chemical multisensory nodes in this setting.

In table 1 we also show results obtained by neglecting information coming from environmental sensors (RH and T sensors). Surprisingly, they show that, notwithstanding the performed datasheet temperature correction procedure, environmental conditions readings still conveyed significant information for reducing estimation error. In fact, multivariate calibration including $\mathrm{RH}$ and $\mathrm{T}$ readings always perform better than the corresponding, same architecture, environmentally unaware system.

In Table 2, we additionally report similar conditions occurring for estimations of other target gases including $\mathrm{O}_{3}$ and $\mathrm{NO}_{\mathrm{x}}$. For all target gases, dynamic multivariate calibration with TDNN always obtain best scores with respect to static multivariate approach (FFNN) and static nonlinear univariate approach obtained feeding a FFNN only with target gas related sensor response.

From a visualization point of view, Fig.7 show the improved correlation among $\mathrm{NO}_{2}$ true values and multivariate estimations with respect to raw $\mathrm{NO}_{2}$ sensor data correlation plot (see Fig.2). Moreover, in Fig.8, we show an example of stochastic estimation of $\mathrm{NO}_{2}$ concentration performed by the TDNN architecture. It shows the precise estimations outputted by the network as well as the confidence intervals as computed with the approach depicted in the previous 
sections. With the proposed architecture each estimation is completed by the confidence interval that is associated with the estimated value.

Finally, in Fig.9 and Fig.10, we address the capability of DNN to follow significantly fast transient with limited precision losses. In particular, in Fig.10, we depict the values of the mean absolute errors ( $y$ axis) obtained partitioning the test set with respect to the target gas absolute first derivative value approximation $(x$ axis $)$ :

$$
\frac{|\Delta y(t)|}{\Delta t}=\frac{|y(t+\Delta t)-y(t)|}{\Delta t}=\frac{\left|y\left(t_{i+1}\right)-y\left(t_{i}\right)\right|}{\Delta t}
$$

with $t_{i} \in\left[t_{0}, T\right], i=1, \ldots, n, n=$ number of samples in the test set, $y(t)=$ target gas concentration $\left(\mathrm{NO}_{2}\right.$, in our case). We compare the static FFNN and the TDNN dynamic network response to rapid transients occurring at different absolute first derivative values. It can be observed that the TDNN is significantly more accurate than the FFNN in following the high speed variation of the target phenomena $\left(\mathrm{NO}_{2}\right.$ concentration). In particular, the faster is the variation of the target variable, the more significant is the enhancement in concentration estimation accuracy that can be achieved by this dynamic architecture.
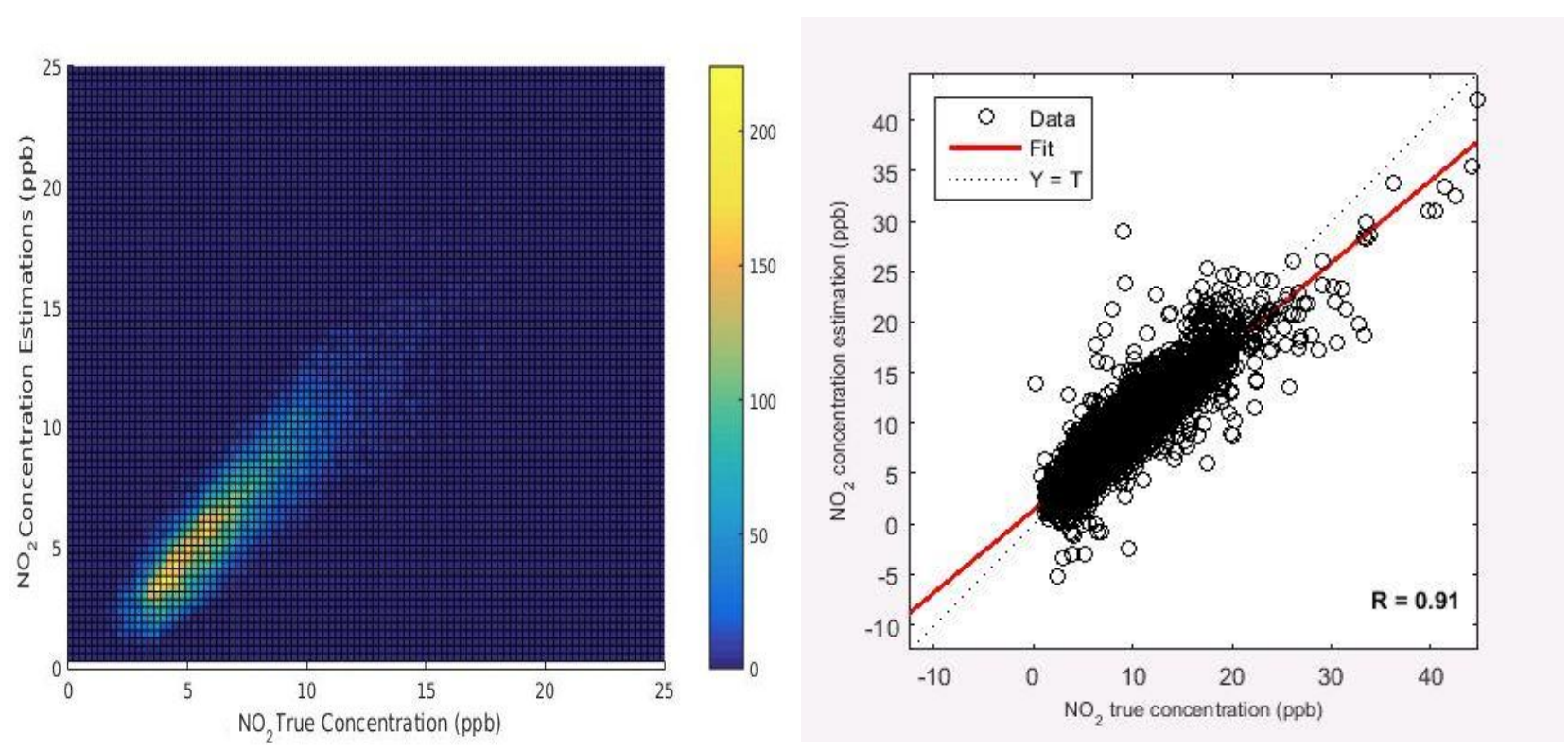

Fig. 7: $\mathrm{NO}_{2}$ concentration estimation versus ground truth correlation plot (TDNN). The picture shows the compact distribution of estimations around true concentrations (left) and the high correlation rate $(R=0.91)$ among them (right). Comparing these results with Figure 2(a), the improvement wrt the uncalibrated sensor reading is evident. On the left, color indicates number of occurrences of the $\left(\mathbf{y}, \mathbf{y}^{\prime}\right)$ couple. 


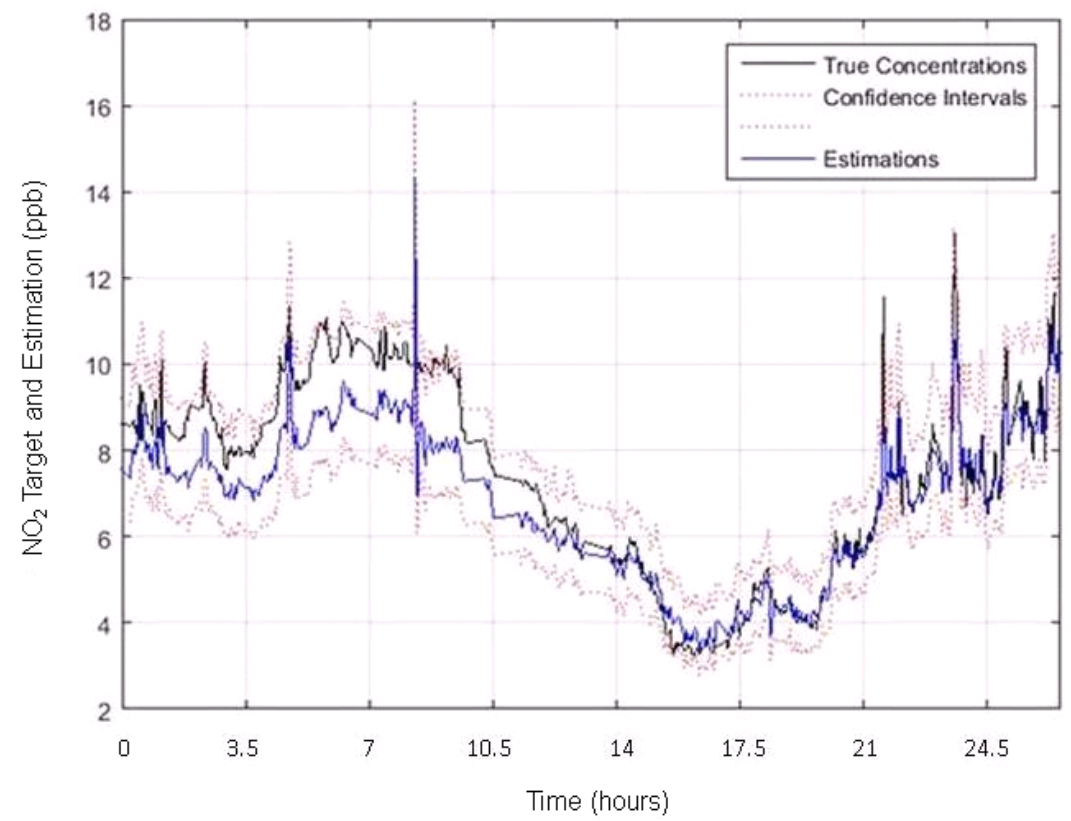

Fig. 8: $\mathrm{NO}_{2}$ concentration estimation performed by TDNN over a temporal subset of test set data $(26 \mathrm{hrs})$ with confidence intervals.

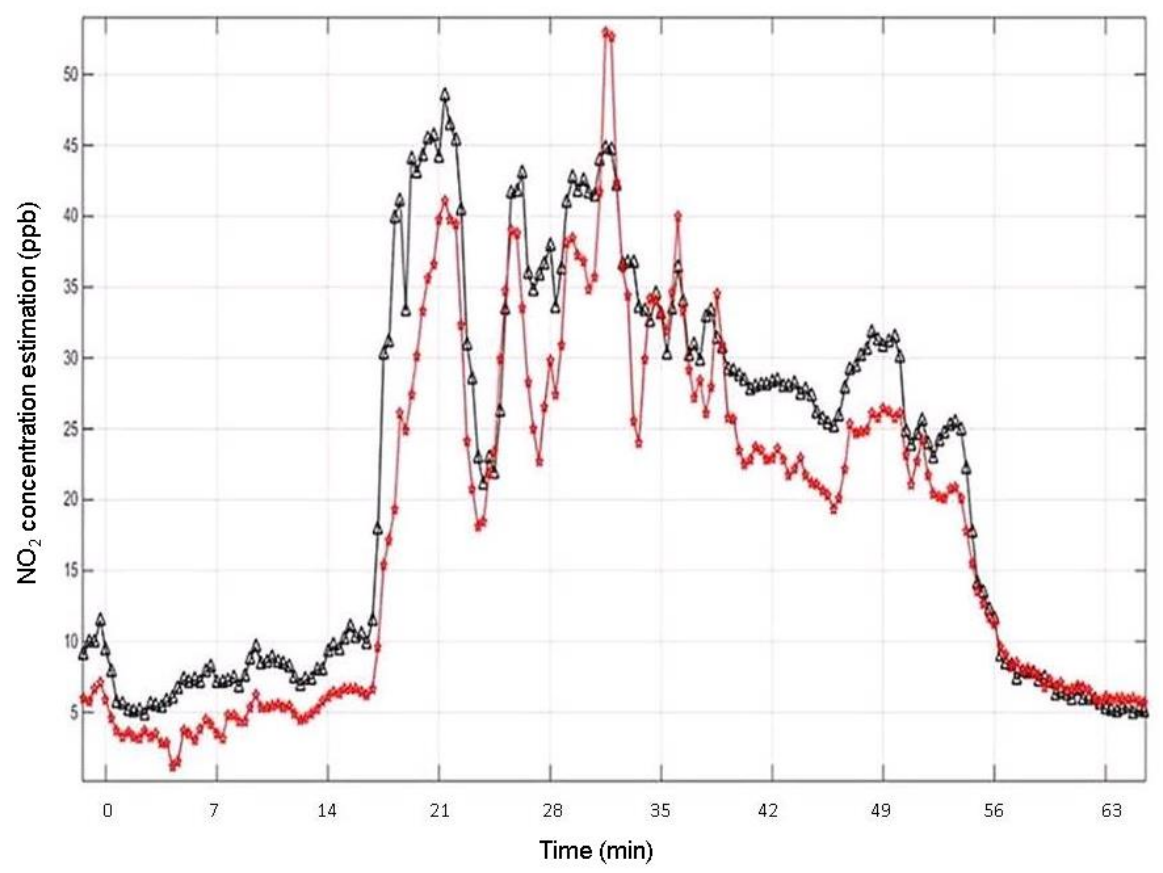

Fig. 9: The network exhibits a very quick response to very rapid transient that challenges the sensors dynamic characteristics (black= true concentration, red $=$ TDNN estimation). 


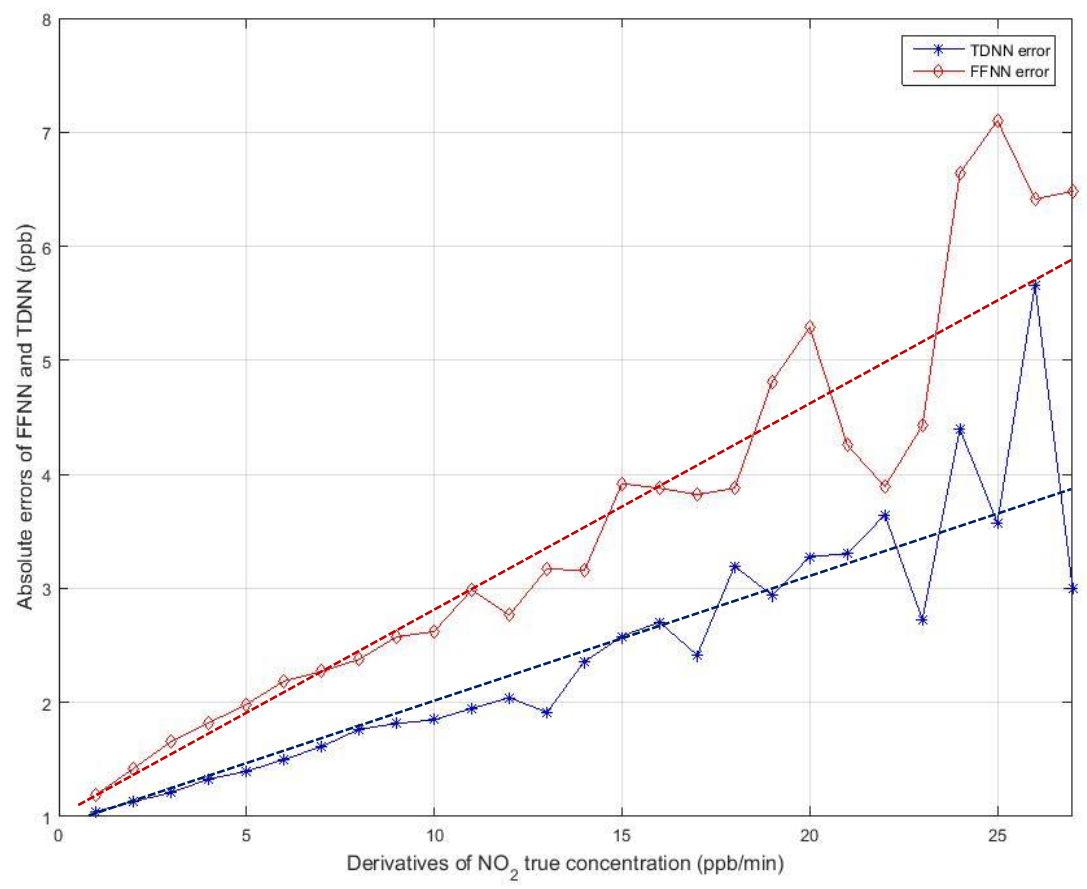

Fig. 10: Comparison of average absolute error distribution trends given the absolute derivative of the $\mathrm{NO}_{2}$ target gas concentration (ppb/min) for dynamic and static network. The TDNN network is capable of achieving a significant reduction of the estimation error wrt the static FFNN network. This behaviour is enhanced when the target gas concentration changes quickly and high values of absolute derivative can be measured.

To this regard, it can be observed that, TDNN absolute error is always smaller than FFNN error, but the differences increase with the absolute derivative values. This confirms that the dynamic model is able to improve the dynamic response to rapid transient with respect to the static model.

TABLE 1: RESULTS OF THE COMPARISON TESTS FOR ESTIMATION OF NO${ }_{2}$ CONCENTRATIONS (TEST SET VALUES).

\begin{tabular}{c|c|c|c|c|c}
\hline Neural Net & Input (Sensors) & Hyper Parameters & MAE $(\mathbf{p p b})$ & MRE & NLPD \\
\hline \multirow{2}{*}{ FFNN } & $\mathrm{NO}, \mathrm{NO}_{2}, \mathrm{O} 3, \mathrm{Rh}, \mathrm{T}$ & $\mathrm{HN}=5$ & $1.50(\mathrm{std}=0.06)$ & 0.25 & $2.15(\mathrm{std}=0.03)$ \\
& $\mathrm{NO}, \mathrm{NO}_{2}, \mathrm{O} 3$ & $\mathrm{HN}=5$ & $1.58(\mathrm{std}=0.04)$ & 0.28 & $1.98(\mathrm{std}=0.01)$ \\
\multirow{3}{*}{ TDNN } & $\mathbf{N O}, \mathbf{N O}_{2}, \mathbf{O 3}, \mathbf{R h}, \mathbf{T}$ & $\mathbf{H N = 5 , ~ T D L = 0 : 6}$ & $\mathbf{1 . 2 7}(\mathrm{std}=\mathbf{0 . 1 0})$ & $\mathbf{0 . 2 2}$ & $\mathbf{1 . 7 8}(\mathrm{std}=\mathbf{0 . 0 5})$ \\
& $\mathrm{NO}, \mathrm{NO}_{2}, \mathrm{O} 3$ & $\mathrm{HN}=5, \mathrm{TDL}=0: 6$ & $1.33(\mathrm{std}=0.05)$ & 0.24 & $1.78(\mathrm{std}=0.02)$ \\
& $\mathrm{NO}, \mathrm{NO}_{2}, \mathrm{O} 3, \mathrm{Rh}, \mathrm{T}$ & $\mathrm{HN}=5, \mathrm{TDL}=0: 6, \mathrm{FDL}=1: 5$ & $1.30(\mathrm{std}=0.15)$ & 0.21 & $1.82(\mathrm{std}=0.10)$ \\
NARXNN & $\mathrm{NO}, \mathrm{NO}_{2}, \mathrm{O} 3$ & $\mathrm{HN}=5, \mathrm{TDL}=0: 6, \mathrm{FDL}=1: 5$ & $1.40(\mathrm{std}=0.10)$ & 0.24 & $1.87(\mathrm{std}=0.21)$ \\
\hline
\end{tabular}

Table 1: Results obtained upon 30 different executions by the proposed architecture by the use of the complete chemical sensor array (+environmental variables). HN $=$ Hidden Number, TDL $=$ Tapped Delay Length and FDL $=$ Feedback Delay Length. Bold values indicates best performance. 
TABLE 2: COMPARISON OF DIFFERENT CALIBRATION METHODOLOGIES FOR ESTIMATION OF $\mathrm{NO}_{2}, \mathrm{O}_{3}, \mathrm{NO}_{\mathrm{X}}$ CONCENTRATIONS OVER TEST SET.

\begin{tabular}{|c|c|c|c|c|c|}
\hline Target & Calibration Methodology & MAE (ppb) & STDERR (ppb) & $\mathrm{CC}$ & MRE (perc) \\
\hline $\mathrm{NO}_{2}$ & Static Univariate & 1.68 & 1.75 & 0.80 & $31 \%$ \\
\hline $\mathrm{NO}_{2}$ & Static Multivariate (FFNN) & 1.50 & 1.69 & 0.84 & $25 \%$ \\
\hline $\mathrm{NO}_{2}$ & Dynamic Multivariate (TDNN) & 1.27 & 1.32 & 0.91 & $22 \%$ \\
\hline $\mathrm{O}_{3}$ & Static Univariate & 8.30 & 6.87 & 0.50 & $90 \%$ \\
\hline $\mathrm{O}_{3}$ & Static Multivariate (FFNN) & 7.90 & 5.21 & 0.82 & $70 \%$ \\
\hline $\mathrm{O}_{3}$ & Dynamic Multivariate (TDNN) & 7.45 & 5.10 & 0.83 & $42 \%$ \\
\hline$N O_{x}$ & Static Univariate & 2.14 & 2.65 & 0.82 & $31 \%$ \\
\hline$N O_{x}$ & Static Multivariate (FFNN) & 1.95 & 2.39 & 0.85 & $29 \%$ \\
\hline$N O_{x}$ & Dynamic Multivariate (TDNN) & 1.37 & 1.61 & 0.94 & $20 \%$ \\
\hline
\end{tabular}

Table 2: Comparison of the results obtained, respectively, with Univariate, Multivariate and Dynamic Multivariate Calibration (TDNN). Bold values indicates best performance.

\section{Conclusions}

In this work, we proposed a dynamic neural networks approach to improve the stochastic estimation of pollutant concentrations by chemical multisensory network devices in real world deployment scenario. Two different dynamic architectures have been trained and tested by relying on a multi-weeks deployment data and compared with state of the art static FFNN approach. A procedure for empirically estimating concentration prediction uncertainty have also been described and used for enhancing prediction semantic value. This procedure is not dependent on the particular computational intelligence approach and could be applied to different calibration approaches. Results, confirming lab based evidences (see [21, 24]), show that dynamic neural networks, by capturing the dynamic behavior of the sensors array, improve the results obtainable by static neural networks over on field recorded data. This improvement affect both accuracy and uncertainty estimations. This improvement may prove decisive in meeting the Data Quality Objective for indicative measurements as expressed by EC 2008 Air Quality directive (see Table 2 for $\mathrm{NO}_{2}$ and $\mathrm{NO}_{\mathrm{x}}$ ). This on field validation is shown, to the best of our knowledge, for the first time in literature. Moreover, Dynamic on field calibration show up as a possible approach to novel issues emerging in mobile and pervasive air quality monitoring deployments with regards to rapid transients. In these cases, our results show that the performance advantage is particularly evident and actually increase when the target gas concentration is rapidly changing. As a secondary result, our data suggests that sensor readings environmental correction procedures for electrochemical sensors should be further improved in order to completely remove the residual environmental effects on sensor responses that we indirectly measured. It is worth to note that this has been achieved with a relatively simple architecture that has an interestingly efficient knowledge representation allowing for on board implementations. Performance analysis on long term deployments are now under evaluation and future works will focus on the effects of sensor dynamic analysis in drift counteraction capabilities of nonlinear on field calibration approaches.

\section{Acknowledgements}

This work has been supported by an STSM (Short Term Scientific Mission) grant from COST Action TD1105 EuNetAir.

\section{References}

[1] EuNETAir COST Action TD1105 Monitoring Progress Reports, 2012-2015, available on www.eunetair.it action website.

[2] T.J. Lyons, W.D. Scott, Principles of Air Pollution Meteorology, Belhaven Press, London, 1990.

[3] B.J. Alloway, D.C. Ayres, Chemical Principles of Environmental Pollution, Blackie Academic \& Professional, London, 1993.

[4] P. Bloomfield, J.A. Royle, L.J. Steiberg, Q. Yang, Accounting for meteorological effects in measuring urban ozone levels and trends, Atmospheric Environment, 30 (1996) 3067-3077. 
[5] S. Marco, A. Gutiérrez-Gálvez, Signal and data processing for machine olfaction and chemical sensing: a review, IEEE Sens. J., 12(11) (2012) 3189-3214.

[6] S. De Vito, M. Piga, L. Martinotto, G. Di Francia, CO, NO2 and NOx urban pollution monitoring with on-field calibrated electronic nose by automatic Bayesian regularization, Sensors and Actuators B, 143 (2008) 182-191.

[7] F. Röck, N. Barsan, U. Weimar, Electronic nose: current status and future trends, Chem. Rev., 108(2) (2008) 705-725.

[8] L. Spinelle, M. Gerboles, M.G. Villani, M. Aleixandre, F. Bonavitacola, Field calibration of a cluster of low-cost available sensors for air quality monitoring. Part A: ozone and nitrogen dioxide, Sens. And Act. B: Chem., 215, (2015), pp. 249-257.

[9] M.Schweizer-Berberich, J. Goppert, A. Hielermann, J. Mitrovics, U. Weimar, W. Rosenstiel. W. Gopel, Application of neural network systems to the dynamic response of polymer-based sensor arrays, Sensors and Actuators B, 26-27 (1995) 232-236.

[10] Monroy, J. G., Lilienthal, A., Blanco, J. L., González-Jimenez, J., \& Trincavelli, Calibration of MOX gas sensors in open sampling systems based on Gaussian Processes, In Sensors, IEEE, (2012) 1-4.

[11] M. Trincavelli, S. Coradeschi, A. Loutfi, Odour classification system for continuous monitoring applications, Sens. Actuators B: Chem., 139(2) (2009) 265-273.

[12] A. Perera, T. Yamanaka, A. Gutiérrez-Gálvez, B. Raman, R. Gutiérrez-Osuna, A dimensionality-reduction technique inspired by receptor convergence in the olfactory system, Sensors and Actuators B: Chem. 116(1) (2006) 17-22.

[13] E. Llobet, O. Gualdrón, M. Vinaixa, N. El-Barbri, J. Brezmes, X. Vilanova, B.Bouchikhi, R. Gómez, J. Carrasco, X. Correig, Efficient feature selection for mass spectrometry based electronic nose applications, Chemometr. Intell. Lab. Syst. 85(2) (2007) 253-261.

[14] I. Rodriguez-Lujan, R. Huerta, C. Elkan, C.S. Cruz, Quadratic programming feature selection, J. Mach. Learn. Res. 11 (2010) $1491-1516$.

[15] J.S. Murguía, A. Vergara, C. Vargas-Olmos, T.J. Wong, J. Fonollosa, R. Huerta, Two-dimensional wavelet transform feature extraction for porous silicon chemical sensors, Anal. Chim. Acta 785 (2013) 1-15.

[16] M.K. Muezzinoglu, A. Vergara, R. Huerta, N. Rulkov, M.I. Rabinovich, A.Selverston, H.D. Abarbanel, Acceleration of chemo-sensory information processing using transient features, Sens. Actuators B: Chem. 137(2) (2009) 507-512.

[17] R. Gutierrez-Osuna, Pattern analysis for machine olfaction: a review, IEEE Sens.J. 2(3) (2002) 189-202.

[18] S. J. Chen, D.C. Hovde, K.A. Peterson, A.W. Marshall, Fire detection using smoke and gas sensors, Fire Saf. J. 42(8) (2007) $507-515$.

[19] A. Pardo, S. Marco, J. Samitier, Nonlinear inverse dynamic models of gas sensing systems based on chemical sensor arrays for quantitative measurements, IEEE Trans. Instrum. Meas., 47(3) (1998) 644-651.

[20] M. Pardo, G. Faglia, G. Sberveglieri, M. Corte, F. Masulli, M. Riani, A time delay neural network for estimation of gas concentrations in a mixture, Sensors and Actuators B: Chem., 65(1) (2000) 267-269.

[21] De Vito, S., Castaldo, A., Loffredo, F., Massera, E., Polichetti, T., Nasti, I., Di Francia, G., Gas concentration estimation in ternary mixture with room temperature operating sensor array using tapped delay neural networks, Sensors and Actuators B, 124 (2007) 309-316.

[22] S. De Vito, E. Massera, M. Piga, L. Martinotto, G. Di Francia, On field calibration of an electronic nose for benzene estimation in an urban pollution monitoring scenario, Sensors and Actuators B: Chem., 129(2) (2008) 750-757.

[23] C. Di Natale, S. Marco, F. Davide, A. D'Amico, Sensor-array calibration time reduction by dynamic modelling, Sens. Actuators B: Chem., 25(1) (1995) 578-583.

[24] S. Sheik et al. Continuous Prediction in Chemoresistive Gas Sensors Using Reservoir Computing, Procedia Engineering, 87 (2014) $843-846$.

[25] J. Fonollosa, S. Sheik, R. Huerta, S. Marco, Reservoir computing compensates slow response of chemosensor arrays exposed to fast varying gas concentrations in continuous monitoring, Sensors and Actuators B: Chem., 2015.

[26] Mead, M. I., Popoola, O. A. M., Stewart, G. B., Landshoff, P., Calleja, M., Hayes, M., Lewis, A., The use of electrochemical sensors for monitoring urban air quality in low-cost, high-density networks, Atmospheric Environment, 70 (2013) 186-203.

[27] O3B4 Ozone Sensor 4-Electrode, data sheet, http://www.alphasense.com/WEB1213/wp-content/uploads/2013/11/O3B4.pdf.

[28] NO2B4 Nitrogen Dioxide, 4_Electrode, Data sheet, http://www.alphasense.com/WEB1213/wp-content/uploads/2013/11/NO2B4.pdf.

[29] C.M. Bishop, Pattern Recognition and Machine Learning, Springer Science, (2006) ISBN 0-387-31073-8.

[30] L. Medsker, L. C. Jain, Recurrent Neural Networks: Design and Applications, CRC Press (1999).

[31] J. Fonollosa, L. Fernández, R. Huerta, A. Gutiérrez-Gálvez, S. Marco, Temperature optimization of metal oxide sensor arrays using mutual information, Sensors and Actuators B: Chem., 187 (2013) 331-339.

[32] J. Fonollosa, I. Rodriguez-Lujan, A.V. Shevade, M.L. Homer, M.A. Ryan, R. Huerta, Human activity monitoring using gas sensor arrays, Sensors and Actuators B: Chem., 199 (2014) 398-402.

[33] F.A. Davide, C.D. Natale, A. D’Amico, A. Hierlemann, J. Mitrovics, M. Schweizer, U. Weimar, W. Göpel, S. Marco, A. Pardo, Dynamic calibration of QMB polymer-coated sensors by Wiener kernel estimation, Sens. Actuators B: Chem., 27(1) (1995) 275-285.

[34] S. Marco, A. Pardo, F.A. Davide, C.D. Natale, A. D’Amico, A. Hierlemann, J. Mitrovics, M. Schweizer, U. Weimar, W. Göpel, Different strategies for the identification of gas sensing systems, Sens. Actuators B: Chem., 34(1) (1996) 213-223.

[35] J. Samitier, J. Lopez-Villegas, S. Marco, L. Camara, A. Pardo, O. Ruiz, J. Morante, A new method to analyse signal transients in chemical sensors, Sens. Actuators B: Chem., 18(1) (1994) 308-312.

[36] E. Llobet, J. Brezmes, X. Vilanova, J.E. Sueiras, X. Correig, Qualitative and quantitative analysis of volatile organic compounds using transient and steady-state responses of a thick-film tin oxide gas sensor array, Sens. Actuators B: Chem., 41(1) (1997) 13-21.

[37] R. Gutierrez-Osuna, Pattern analysis for machine olfaction: a review, IEEE Sens. J., 2(3) (2002) 189-202.

[38] P. Althainz, J. Goschnick, S. Ehrmann, H. Ache, Multisensor microsystem for contaminants in air, Sens. Actuators B: Chem., 33(1) (1996) $72-76$.

[39] L. Zhang, F. Tian, S. Liu, J. Guo, B. Hu, Q. Ye, L. Dang, X. Peng, C. Kadri, J. Feng, Chaos based neural network optimization for concentration estimation of indoor air contaminants by an electronic nose, Sens. Actuators A: Phys., 189 (2013) 161-167.

[40] M.T. Hagan, M.B. Menhaj, Training feedforward networks with the marquardt algorithm, IEEE Trans. Neural Networks, 5 (1994) $989-993$.

[41] Monroy, J. G., Lilienthal, A. J., Blanco, J. L., Gonzalez-Jimenez, J., \& Trincavelli, M., Probabilistic gas quantification with MOX sensors in Open Sampling Systems - A Gaussian Process approach, Sens. Actuators B: Chem., 188 (2013) 298-312.

[42] S. Vembu, A. Vergara, M. K. Muezzinoglu, R. Huerta, On time series features and kernels for machine olfaction, Sensors and Actuators B: Chemical, Volume 174, November 2012, Pages 535-546, ISSN 0925-4005, http://dx.doi.org/10.1016/j.snb.2012.06.070.

[43] J. Quinonero-Candela, C. E. Rasmussen, F Sinz, O. Bousquet, B. Scholkopf, Evaluating Predictive Uncertainty Challenge, Lecture Notes in Computer Science, Vol. 3944, 2006, pp. 1-27 


\section{Biographies}

Elena Esposito received her Degree in Mathemathics (cum laude) in September 2008 and her PhD in Numerical Analysis in 2011 both from University of Salerno. She received a fellowship from University of Salerno in 2013, for research activities related to the Electronic Money System and Multi-Channel Value-Added Services. In 2015, She joined ENEA with a postdoctoral research fellowship. Her research interests include Numerical methods for Ordinary Differential Equations, Machine Learning techniques in the artificial olfaction framework. She is currently involved in national and international projects dealing with application of intelligent sensing methodologies in air and water quality monitoring.

Saverio De Vito received his degree in Computer Engineering from University of Naples "Federico II" in 1998 and his Ph.D. in Information Engineering from University of Cassino. Previously involved in biomedical image processing, from 1999 to 2004 he was R\&D technical manager for satellite based telemedicine, and distance learning projects in a software house. In June 2004, he joined ENEA, as a researcher. His research interests include artificial olfaction, wireless and intelligent sensing, air quality monitoring, statistical pattern recognition, and computer aided diagnosis. Involved in several national and international research projects and member of several committee in cooperative research efforts, He is author of more than 40 scientific contributions. Since 2005 he is adjunct professor of Computer Science and Parallel Computing at University of Cassino and South Lazio.

Maria Salvato received her Degree in Mathemathics (cum laude) in 2008 and her PhD in Mathematical Analysis in 2011 both from University of Salerno. She was granted a research fellowhip from University of Cassino in 2014. In 2015 She joined ENEA with a potdoctoral research fellowship. Her research interests include Elliptic Partial Differential Equation solving methods and pattern recognition in the artificial olfaction framework. She is currently involved in national and international projects (FP7) dealing with application of intelligent sensing methodologies in aerospace industry.

Vivien Bright is a Post-doctoral researcher in Atmospheric Science in the Centre for Atmospheric Science, Department of Chemistry, at the University of Cambridge. Following a BSc in Marine Geography at Cardiff University, Vivien undertook an MSc in Applied Meteorology at the University of Reading. After completion of her MSc in 2006, Vivien joined the Met Office and trained as a weather forecaster. In 2008, Vivien began a Ph.D. in Atmospheric Science at the University of Birmingham, supervised by Dr William Bloss and Dr Xiaoming Cai. Her Ph.D. research involved developing a Large Eddy Simulation (LES) model to include a more detailed chemical reaction scheme, and to use this enhanced model to investigate the combined effects of mixing and chemical reactions upon urban air quality. Vivien joined the University of Cambridge in 2012 working with Professor Rod Jones as a research associate as part of the small sensors group. Her research interests include studying atmospheric composition in urban areas, boundary layer meteorology and determining personal exposure.

Olalekan (Lekan) Popoola is currently a Post-doctoral researcher in Atmospheric Science at the Centre for Atmospheric Science, Department of Chemistry, at the University of Cambridge. He has a BSc in Pure and Applied Chemistry at Ladoke Akintola University of Technology, Ogbomoso, Nigeria. In 2008, he obtained an MSc in Physical Chemistry at the University of Ibadan, Nigeria. Lekan proceeded to the University of Cambridge in 2009 for his Ph.D. Atmospheric Science at the Center for Atmospheric Science, Chemistry Department under the supervision of Professor Roderic Jones. His Ph.D. research involved studies of urban air quality using electrochemical based sensor instruments. His main work involved characterization, deployment and data analysis of outdoor air quality using novel portable low-cost air quality sensor. He has since been working in Rod Jones group since completing his $\mathrm{PhD}$ in 2012 as a research associate as part of the small sensors group. Her research interests include studying atmospheric composition in urban areas, air quality monitoring/modelling, characterization of air quality instruments as well as network data management and analysis. Lekan is also a senior member of Queens' College Cambridge.

Roderic (Rod) Jones is Professor of Atmospheric Science in the Centre for Atmospheric Science, Department of Chemistry, at the University of Cambridge. He obtained his first degree in Physics and a D. Phil. in Atmospheric Physics, both at the University of Oxford. He moved to Cambridge in 1990, after spending 5 years at the UK Meteorological Office where he was involved with studies of the Antarctic ozone 'hole'. His research interests are the structure and composition of the atmosphere, covering a wide range of issues from local air quality to global climate change, with a strong emphasis on atmospheric measurements. He has a strong background in novel spectroscopic techniques including DOAS, CRDS and LIDAR, and in recent years he has pioneered the development of low cost ultra-small gas sensors and sensor networks for atmospheric studies. His work on sensor networks and sensor network systems (including chemical sensors, multi-node data transmission, network management and sensor calibration, and tools for data mining and display) has led to major research and development programs extending across air quality, exposure and human health, and greenhouse gas emissions. Rod Jones is also a Fellow of Queens' College Cambridge where he teaches all aspects of undergraduate physical chemistry. 